Revista Calidad en la Educación Superior

Programa de Autoevaluación Académica

Universidad Estatal a Distancia

ISSN 1659-4703

Costa Rica

revistacalidad@uned.ac.cr

\title{
IMPLICACIONES DE LA CULTURA ORGANIZACIONAL DE INSTITUCIONES DE EDUCACIÓN SUPERIOR EN LA IMPLANTACIÓN DE SISTEMAS DE GESTIÓN DE LA CALIDAD CON RESPONSABILIDAD SOCIAL
}

\section{IMPLICATIONS OF ORGANIZATIONAL CULTURE OF HIGHER EDUCATION SYSTEMS IN THE IMPLEMENTATION OF QUALITY MANAGEMENT WITH SOCIAL RESPONSIBILITY}

\begin{abstract}
Categoría del artículo: noción de cultura organizacional
Julio Márquez Rodríguez

Sergio Ochoa Jiménez

Beatriz Ochoa Silva

Instituto Tecnológico de Sonora, México
\end{abstract}

Volumen 4, número 2

Noviembre 2013

pp. $48-128$

Recibido: agosto, 2013

Aprobado: noviembre, 2013

\footnotetext{
*Es maestro; además es estudiante del Programa de Doctorado en Planeación Estratégica para la Gestión del Desempeño en el Instituto Tecnológico de Sonora, México. Correo electrónico: juliomarquez1@hotmail.com

"Es Doctor y profesor titular del Programa de Doctorado en Planeación Estratégica para la Gestión del Desempeño en el Instituto Tecnológico de Sonora, México. Correo electrónico: sergio.ochoa@itson.edu.mx

**** Doctora y profesora titular del Programa de Doctorado en Planeación Estratégica para la Gestión del Desempeño en el Instituto Tecnológico de Sonora, México. Correo electrónico: beatriz.ochoa@itson.edu.mx
} 


\section{Resumen}

Los rasgos de la cultura organizacional de las instituciones de educación superior juegan un papel preponderante para facilitar la implementación de propuestas de mejora. El presente artículo muestra los resultados de un estudio de cultura organizacional en una universidad mexicana que se prepara para la implementación de un sistema de gestión de la calidad con responsabilidad social, con la finalidad de reconocer, a través de la adecuación de un modelo de diagnóstico y la aplicación de herramientas de recopilación de datos, cuáles son los elementos culturales que favorecen o limitan dicha implementación. A partir de los resultados se propone un conjunto de estrategias para gestionar los elementos culturales y alinearlos en función de los objetivos estratégicos de la organización.

Palabras claves: cultura organizacional, educación superior, calidad, responsabilidad social

\section{Abstract}

The features of the organizational culture of higher education institutions play an important role to facilitate the implementation of proposals for improvement. This article shows the results of a study of organizational culture on a MexicanUniversity preparing for the implementation of a quality management social responsibility, in order to recognize, through the adaptation of a model of diagnosis and implementation of data collection tools, what are the cultural elements that favor or limit such implementation. From the results, we propose a set of strategies to manage cultural elements and align them according to the organization's strategic objectives.

Keywords: Organizational Culture, Higher Education, Quality, Social Responsibility

\section{Introducción}

Para cualquier organización la habilidad para adaptarse a los constantes cambios que supone el entorno global, la aparición de nuevas tendencias sociales y la permanente evolución tecnológica, constituye un factor de vital importancia para mantener e incrementar su competitividad a lo largo del tiempo. 
Las organizaciones requieren un conjunto de condiciones que sin duda dependen en gran medida de las competencias de los colaboradores que las conforman. En ese sentido, la efectividad de los integrantes de una organización, y por tanto la competitividad de la propia organización, está directamente relacionada con las políticas, las prácticas, las normas y los supuestos compartidos por todos los miembros de la misma, en un conglomerado de elementos que se ha denominado la cultura organizacional.

Para contextualizar la importancia de la cultura organizacional es preciso identificar primero la cultura, que se reconoce como el conjunto de patrones compartidos heredados por la sociedad a la que pertenece, que sirven de guía de comportamiento, generan pautas de conducta y generalmente le dan al individuo sentido de pertenencia e identidad. De acuerdo con Toca y Carrillo (2009), estos patrones habitan en la mente de los individuos y se toman del ambiente y el contexto en el que se desarrollan.

El desarrollo de la cultura es lo que diferencia a las sociedades humanas de otras sociedades de especies animales. De la misma forma, la cultura de una organización le permite diferenciarse de cualquier otra y brindar a sus miembros una mayor identificación y sentido de pertenencia.

Herriegel y Slocum (2009) consideraban que, a diferencia de la misión y la visión -cuyos enunciados suelen existir de forma escrita-, la cultura organizacional, además de no tener un registro formal, constituye la personalidad de la organización, y su identificación y conocimiento resultan determinantes en la expectativa de incrementar la lealtad, la productividad, la rentabilidad y la competitividad de una organización.

Por lo tanto, se puede decir que la cultura de la organización constituye un factor determinante en el mejoramiento del desempeño, ya que se relaciona no solo con los resultados y la eficiencia de la organización, sino también con la identidad, el 
compromiso y la calidad del trabajo de sus colaboradores. Por ello, es preciso identificar los principales componentes de la cultura organizacional, comprenderlos y gestionarlos, con la intención de propiciar las condiciones para favorecer las actitudes y conductas que generen un ambiente adecuado para el logro de los objetivos institucionales.

Si bien existe un amplio conjunto de definiciones de la cultura organizacional y diferentes posturas para su análisis, derivadas de la diferencia en la óptica usada para su interpretación, conceptualmente existe un consenso general en identificarla como el conjunto de patrones y significados que comparten los miembros de una organización (Schein 2010). Generalmente, estos patrones generan pautas de conducta, sirven de guía de comportamiento, le proporcionan al colaborador un sentido de pertenencia e identidad con la organización y le permiten a la organización diferenciarse de cualquier otra.

Dado que cualquier aproximación al estudio de la cultura organizacional involucra el estudio de fenómenos sociales, el rango de elementos de la cultura organizacional es variado de acuerdo a las diferentes posturas y los autores.

Sin embargo, Schein (2010) propuso un marco de referencia integrado por tres capas básicas que permiten agrupar los distintos elementos de la cultura organizacional. La primera capa, conocida también como dimensión esencial, la constituyen el conjunto de premisas que impactan contundentemente en la mayoría de pensamientos y acciones de la organización, es decir, los valores, las ideologías, los supuestos y el conocimiento. La segunda capa se integra únicamente por las creencias estratégicas, mismas que se sustentan en las convicciones y certezas de los líderes organizacionales. La tercera capa, conocida como dimensión manifiesta, incorpora todo tipo de prácticas, el lenguaje, los símbolos, las historias, los rituales, las rutinas, los estándares, las convenciones y las costumbres, es decir, la conducta modelada. 


\section{La cultura organizacional y la calidad en las instituciones de educación superior}

El estudio e identificación de las características de la cultura organizacional se ha convertido en una temática de gran interés para el mejoramiento del desempeño de las organizaciones, ya que permite contar con una amplia perspectiva para conocer a la organización de manera profunda, entendiendo su relación con los individuos que la integran y las condiciones de las relaciones con el entorno en el que se desarrolla.Es sabido que no existe organización sin una cultura inherente que le dé identidad y la distinga, al tiempo que guía su actividad y rige las percepciones que de ella tienen sus grupos de interés.

Las instituciones de educación superior (IES), como cualquier organización, tienen manifestaciones culturales que deben analizarse y gestionarse para lograr alinear los elementos de su cultura organizacional al logro de sus objetivos estratégicos.

La educación superior es esencialmente el proceso mediante el cual es posible formar personas con competencias específicas para desarrollar un trabajo a nivel profesional; y además es el medio para detonar el desarrollo económico y social de una comunidad, por lo que de su calidad e integralidad dependerán en gran medida el progreso de la sociedad, el mantenimiento de la convivencia entre individuos y la sustentabilidad de los actuales y futuros estilos de vida.

Tradicionalmente, las IES han tenido propósitos en el ámbito social, como formadoras del capital humano, que impulsaron el desarrollo económico de las sociedades, pero en las últimas décadas se han incorporado a estos propósitos nuevos objetivos orientados a garantizar servicios educativos de buena calidad y con atributos de pertinencia, cobertura y equidad. En este sentido, la adopción de modelos de gestión de la calidad y la responsabilidad social en las IES puede significar una forma de incrementar la transparencia y rendición de cuentas al tiempo, que promuevan el ejercicio de valores éticos, sociales y medioambientales en sus comunidades. Lo anterior no solo con la finalidad de formar profesionistas 
cada vez más competitivos, sino como medidas para hacerle frente a la gran expectativa de promoción del desarrollo social y económico que se tiene de las IES (Barrera, 2007).

Considerando lo anterior, el presente estudio propone la identificación de los principales rasgos de la cultura organizacional en una IES mexicana que pretende implementar un sistema de gestión de la calidad y la responsabilidad social, basada en las normas ISO 9001:2008 e ISO 2600:2010, esto con el objetivo de reconocer cuáles son los elementos culturales que favorecen o limitan la implementación de esta propuesta de mejora y contar con las condiciones para gestionarlos y alinearlos en función de sus objetivos estratégicos.

\section{Elementos metodológicos}

Para llevar a cabo un estudio de la cultura organizacional en una IES es preciso contar con una serie de elementos metodológicos que incluyan un modelo específico para realizar el diagnóstico, la definición del tipo de estudio, los participantes y los instrumentos de recopilación y análisis de datos.

\section{Modelo de diagnóstico.}

Para comenzar el estudio se realiza un análisis de cinco modelos de diagnóstico de la cultura organizacional que incluyen variables relacionadas con la implementación de estrategias de mejora de la calidad en la organización, como el modelo de Martínez y Robles (2009), que destaca la importancia del entendimiento y de la satisfacción de los requisitos de los clientes internos y externos; el modelo de Buenaventura (1995), que se enfoca en el establecimiento de estrategias de motivación del personal para favorecer el cambio cultural que supone la implementación de una gestión de la calidad; el modelo de Hernández, et al (2007), que resalta la importancia del desarrollo de una cultura de calidad en la que existan individuos comprometidos y colaborativos, responsables de la 
satisfacción de sus clientes internos y externos; el modelo de Montes (2012), dirigido a IES que buscan conformar una cultura interna que les permita propiciar condiciones para administrar la calidad del servicio que ofertan; $y$, finalmente, el modelo de Fernández (2002), que logra una adaptación al ámbito escolar de varios modelos teórico-metodológicos sobre la cultura corporativa.

Posteriormente, se realiza una adaptación que integra un conjunto de diez variables fundamentadas no solo en los modelos anteriores, sino en las aportaciones de Maull, Brown y Cliffe (2001) y Robbins y Judge (2009), con lo cual se asegura que el modelo se oriente a la implementación de un sistema de gestión de la calidad eficiente, impregnado de los principios culturales de la IES, es decir, de los rasgos mas relevantes de su cultura organizacional. 


\section{Tabla 1 \\ Modelo de diagnóstico de la cultura organizacional orientado a la implementación de un sistema de gestión de la calidad con responsabilidad social en una IES}

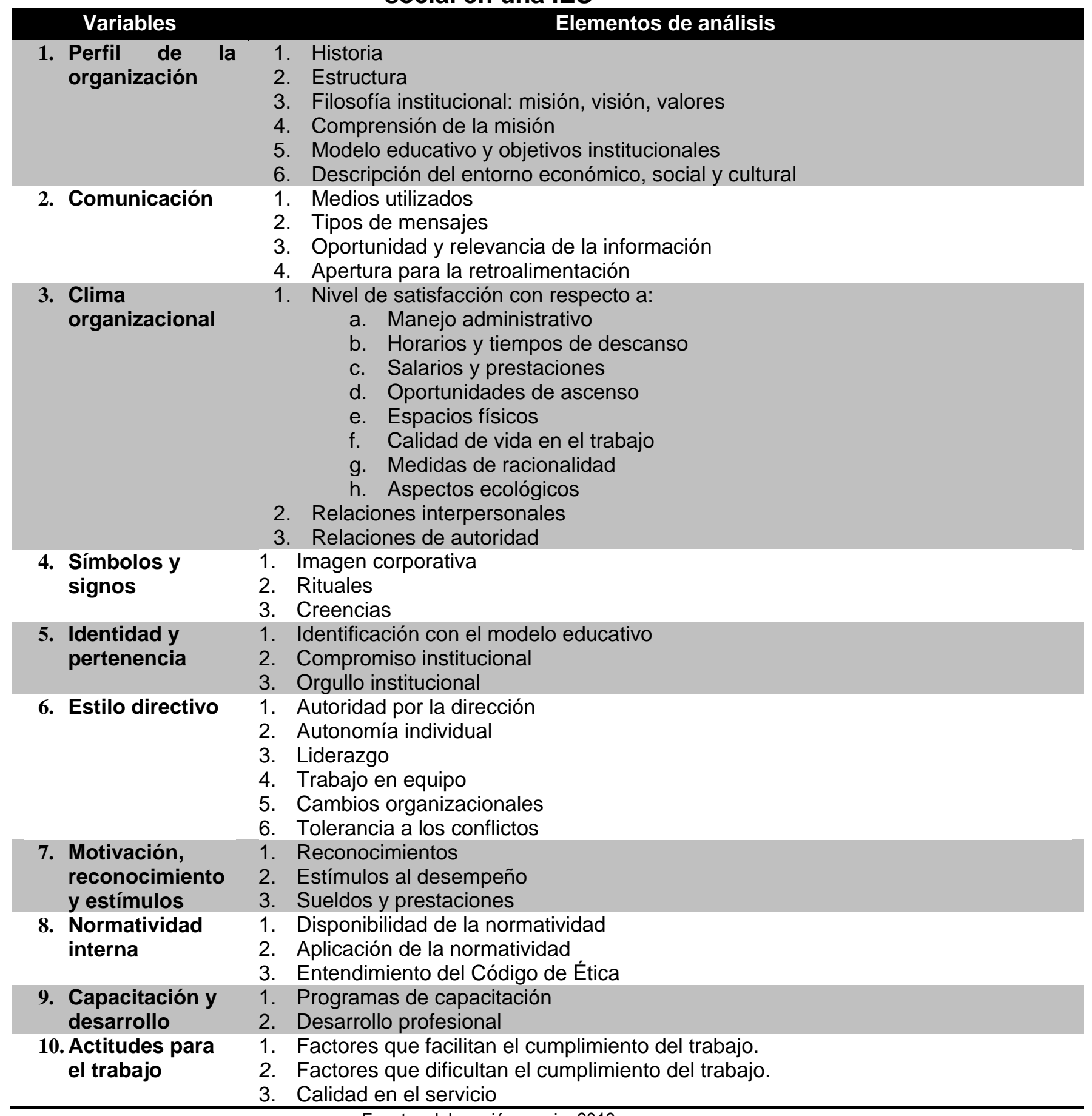

Fuente: elaboración propia, 2013 


\section{Tipo de estudio, instrumentos, participantes y análisis de datos.}

El presente estudio es de tipo descriptivo, y para llevarlo a cabo se diseñaron dos instrumentos: una entrevista estructurada para evaluar la primera variable y una encuesta electrónica para evaluar las restantes variables, integrada por 65 reactivos, 60 con opciones de respuesta con escala tipo Likert, cuya consistencia interna fue validada mediante el cálculo del Coeficiente Alpha de Cronbach con un valor de 0.996, y cinco preguntas abiertas, aplicada a una muestra del $50 \%$ del personal académico y de apoyo a la docencia, de una población total de 121 colaboradores. La muestra fue seleccionada de manera proporcional a la cantidad de ambos tipos de colaboradores. Durante el análisis de datos se utilizó la técnica estadística de la distribución de frecuencias para identificar y clasificar los resultados obtenidos a partir de la encuesta electrónica (Hernández et al, 2008).

\section{Resultados.}

A continuación se describen los resultados del estudio agrupados por categorías del modelo utilizado:

\section{Perfil de la organización.}

La IES seleccionada para el presente estudio es un organismo descentralizado de la administración pública estatal, con personalidad jurídica y patrimonio propios, que tiene por objeto impartir educación superior en los niveles de licenciatura, especialidad, maestría y doctorado, para preparar profesionales con una sólida formación científica y tecnológica y con valores cívicos y éticos, conscientes del contexto nacional en lo económico, político y social; enfocada en potenciar la investigación aplicada y el desarrollo tecnológico pertinentes para el progreso económico y social de la región y de México, difundir el conocimiento y la cultura a través de la extensión universitaria y la formación a lo largo de toda la vida y prestar servicios tecnológicos y de asesoría que contribuyan a mejorar el desempeño de las empresas y otras organizaciones de la región. Cuenta con una misión, una visión, valores institucionales y un Código de Ética que regulan la vida 
Implicaciones de la cultura organizacional de instituciones de educación superior en la implantación de sistemas de gestión de la calidad con responsabilidad social

Julio Márquez Rodríguez, Sergio Ochoa Jiménez, Beatriz Ochoa Silva

universitaria y orientan los esfuerzos del personal hacia el logro de los objetivos institucionales, entre los que destacan por su alineación a la propuesta de mejora: asegurar la aportación social transformadora, incrementar la cobertura con calidad y equidad, desarrollar investigaciones, servicios tecnológicos y de consultoría pertinentes y de alta calidad; y certificar los procesos académicos y de gestión.

\section{Comunicación.}

Para la evaluación de esta variable se consideraron los medios utilizados, el tipo de mensajes, la oportunidad y la relevancia de la información, así como la apertura para la retroalimentación por parte de los colaboradores.

Figura 1

Resultados de la categoría Comunicación

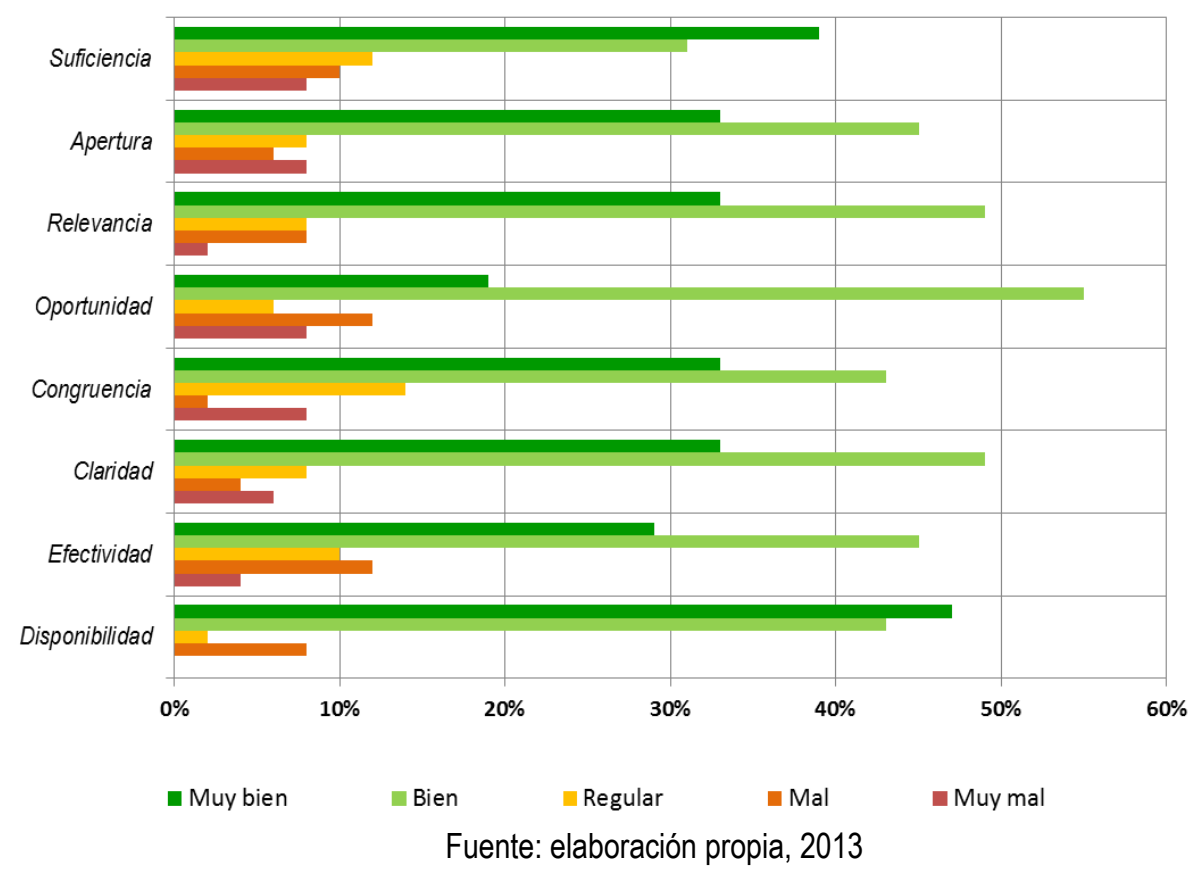

La mayor fortaleza de la organización se encuentra en la suficiencia y disponibilidad de medios y en la claridad de los mensajes de los directivos, así 
Implicaciones de la cultura organizacional de instituciones de educación superior en la implantación de sistemas de gestión de la calidad con responsabilidad social

Julio Márquez Rodríguez, Sergio Ochoa Jiménez, Beatriz Ochoa Silva

como en la contribución de la comunicación al cumplimiento de las funciones asignadas y en la apertura para la retroalimentación.

Por otro lado, es posible identificar áreas de mejora en la efectividad y agilidad de los medios, en la apertura y, especialmente, en la suficiencia de los mecanismos para recopilar las quejas y sugerencias del personal.

\section{Clima organizacional.}

Las principales fortalezas de la organización en cuanto al clima laboral se concentran en la satisfacción con los horarios de trabajo y el descanso, el volumen de trabajo administrativo y las relaciones sociales entre compañeros y con las autoridades.

En relación con las oportunidades de ascenso, las medidas de racionalidad y las ecológicas y la calidad de vida laboral la percepción se inclina hacia la satisfacción. Sin embargo, se aprecian áreas de oportunidad en la mejora de aspectos tales como los salarios, las prestaciones y los espacios físicos, ya que la percepción en este sentido es muy distante de la satisfacción y representa serias debilidades para la organización. 
Implicaciones de la cultura organizacional de instituciones de educación superior en la implantación de sistemas de gestión de la calidad con responsabilidad social

Julio Márquez Rodríguez, Sergio Ochoa Jiménez, Beatriz Ochoa Silva

Figura 2

Resultados de la categoría Clima organizacional

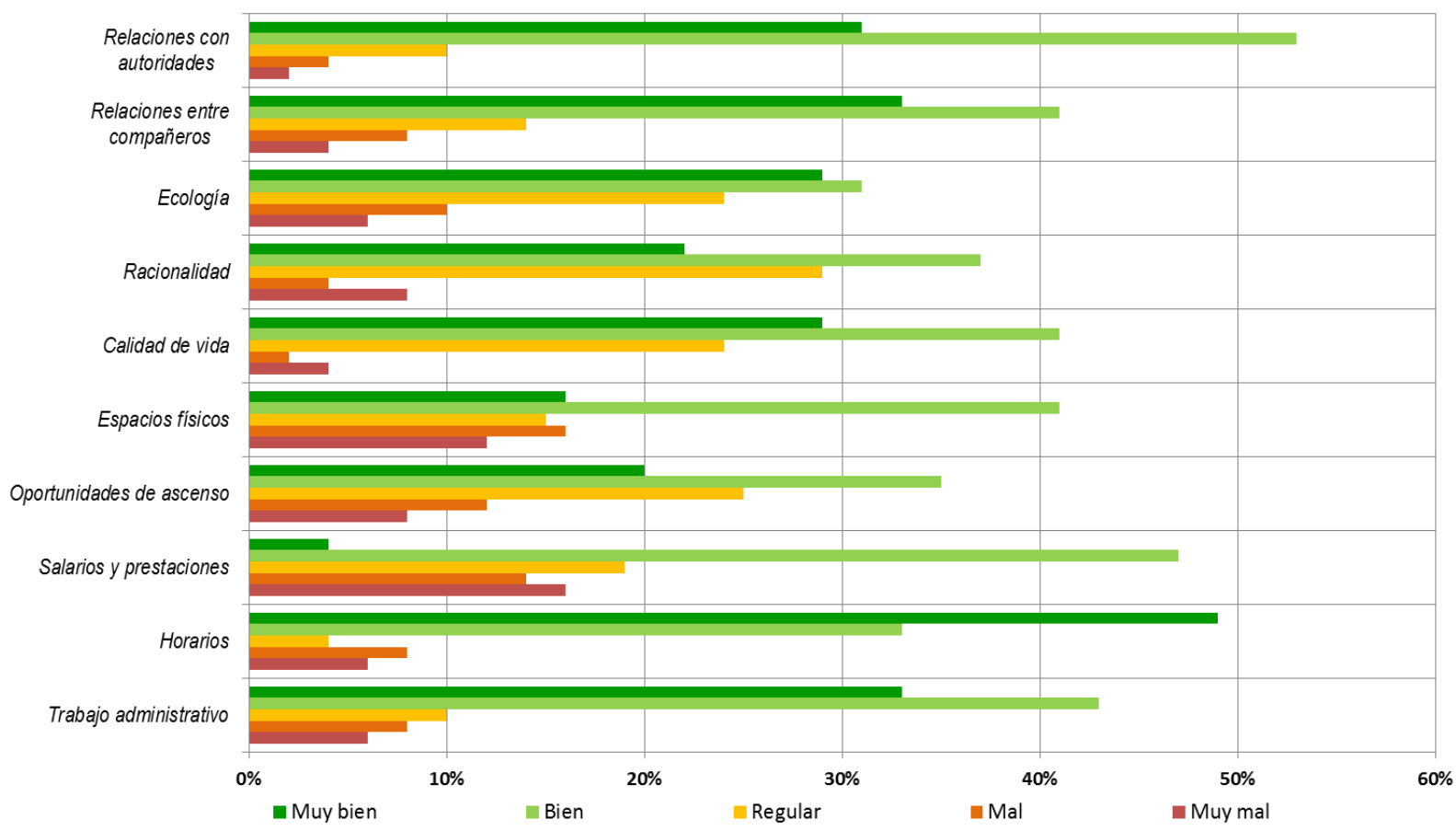

Fuente: elaboración propia, 2013

\section{Símbolos y signos.}

La evaluación respecto a los símbolos y signos, como parte de la cultura organizacional, permite apreciar fortalezas en cuanto a la difusión de la imagen institucional y la utilización de símbolos que la fortalecen, tales como el uniforme, los logotipos y los elementos mercadológicos.

A pesar de que se reconoce parcialmente la existencia de rituales en la organización y que el personal considera que son compartidos, existe una sección importante del personal que opina que no son compartidos ampliamente, lo cual supone una oportunidad para el logro de mayor cohesión.

De igual manera ocurre con las creencias en la organización, ya que, a pesar de que la mayoría opina que son compartidas, una parte importante del personal 
Implicaciones de la cultura organizacional de instituciones de educación superior en la implantación de sistemas de gestión de la calidad con responsabilidad social

Julio Márquez Rodríguez, Sergio Ochoa Jiménez, Beatriz Ochoa Silva

considera que no necesariamente reflejan la convicción por colaborar en el cumplimiento de los objetivos institucionales.

Figura 3

Resultados de la categoría Símbolos y signos

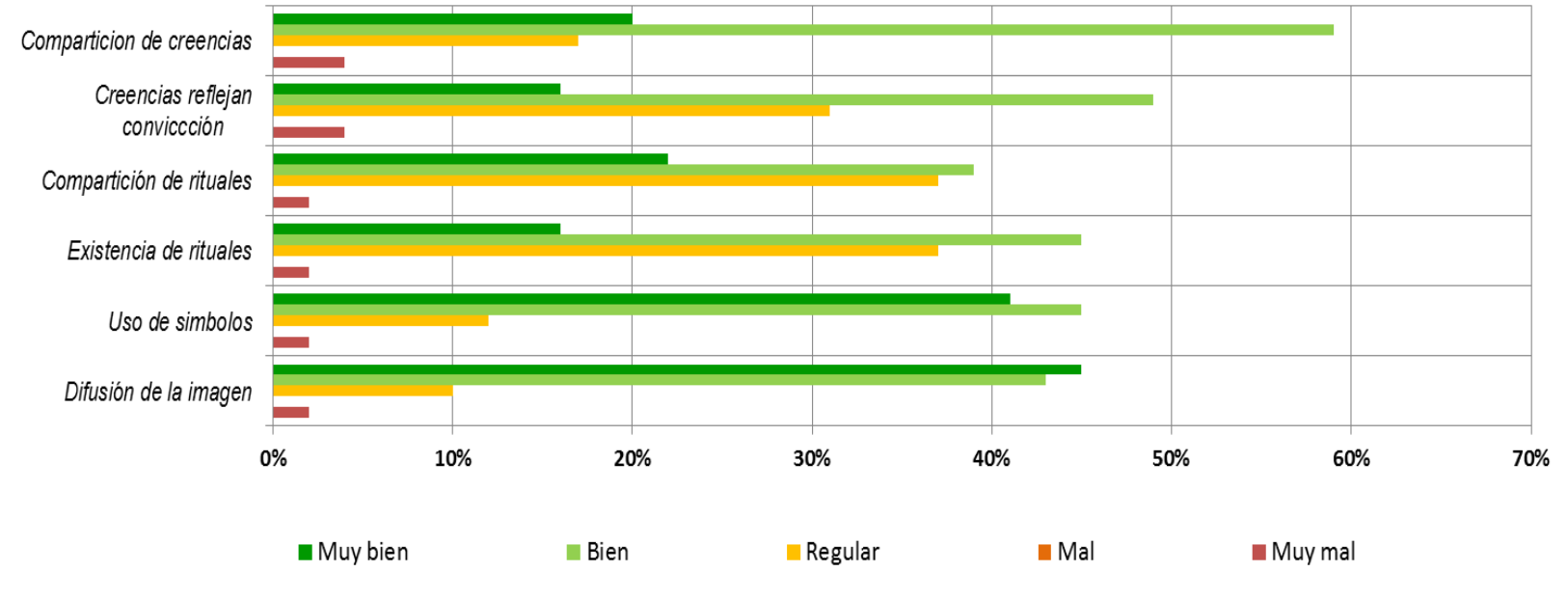

Fuente: elaboración propia, 2013

\section{Identidad y pertenencia.}

En esta categoría se ubican rasgos culturales que se relacionan con la identificación del personal con el modelo educativo, con el compromiso hacia la institución y con el orgullo de pertenecer a la organización. Los resultados permiten concluir que el sentido de pertenencia y el orgullo institucional son fortalezas de la organización, especialmente en cuanto a la contribución del trabajo hacia el cumplimiento de la misión. Específicamente, el orgullo institucional tiene los más altos registros de satisfacción del personal, por lo que representa un elemento de gran utilidad en las estrategias de mejora del desempeño. 
Implicaciones de la cultura organizacional de instituciones de educación superior en la implantación de sistemas de gestión de la calidad con responsabilidad social

Julio Márquez Rodríguez, Sergio Ochoa Jiménez, Beatriz Ochoa Silva

Por otro lado, el sentido de utilidad del trabajo y su alineación al modelo educativo se encuentran bien desarrollados, lo mismo que la disponibilidad para la colaboración; esto permite inferir que es posible establecer estrategias de mejora del desempeño basadas en estas variables de fomento al sentido de pertenencia y orgullo institucional.

Figura 4

Resultados de la categoría Identidad y pertenencia

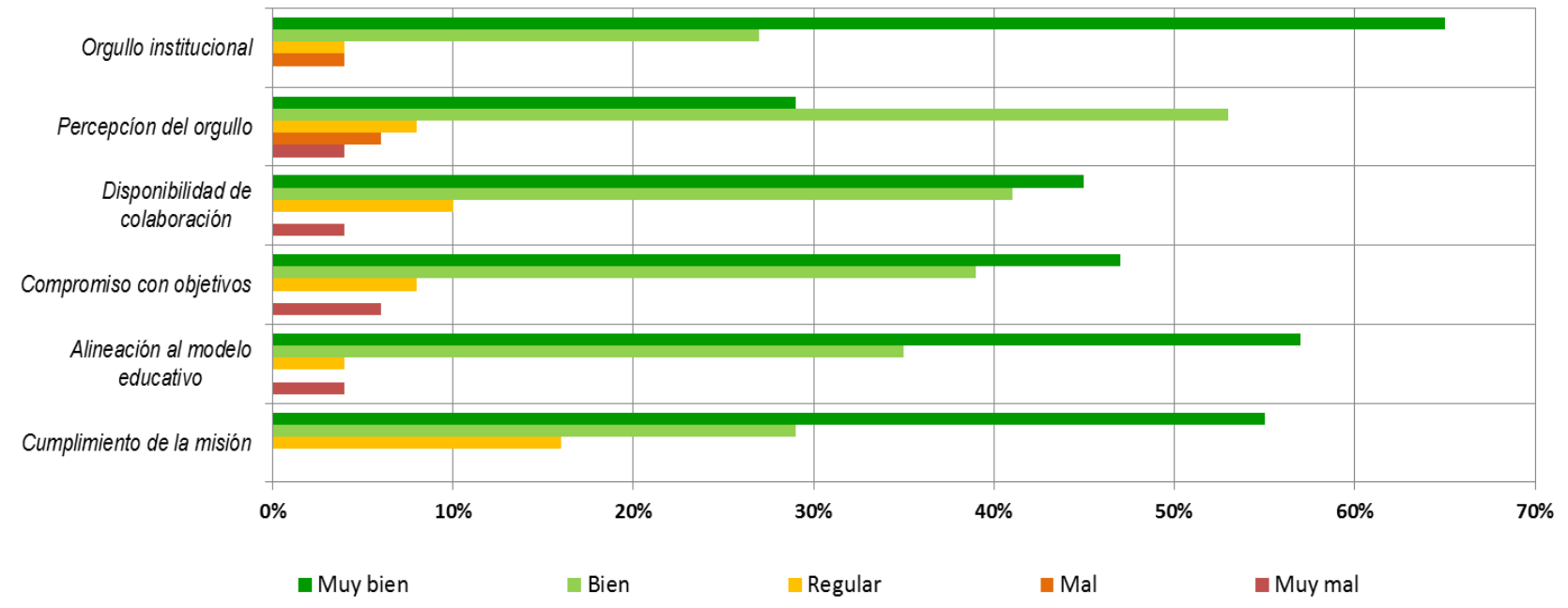

Fuente: elaboración propia, 2013

\section{Estilo directivo.}

Las mayores fortalezas en esta categoría radican en la habilidad directiva para buscar consenso y en la satisfacción de la persona respecto a la percepción que los directivos tienen de ella. La promoción del trabajo en equipo, la motivación para el desempeño, los esfuerzos para lograr la integración del personal y la apertura hacia el cambio e innovación por parte de los directivos son elementos que, si bien no están desarrollados al máximo, muestran relativa tendencia hacia la fortaleza. Las áreas que presentan mayores debilidades se relacionan con la 
Implicaciones de la cultura organizacional de instituciones de educación superior en la implantación de sistemas de gestión de la calidad con responsabilidad social

Julio Márquez Rodríguez, Sergio Ochoa Jiménez, Beatriz Ochoa Silva

oportunidad de la información acerca de los cambios que se llevan en la institución.

Figura 5

\section{Resultados de la categoría Estilo directivo}

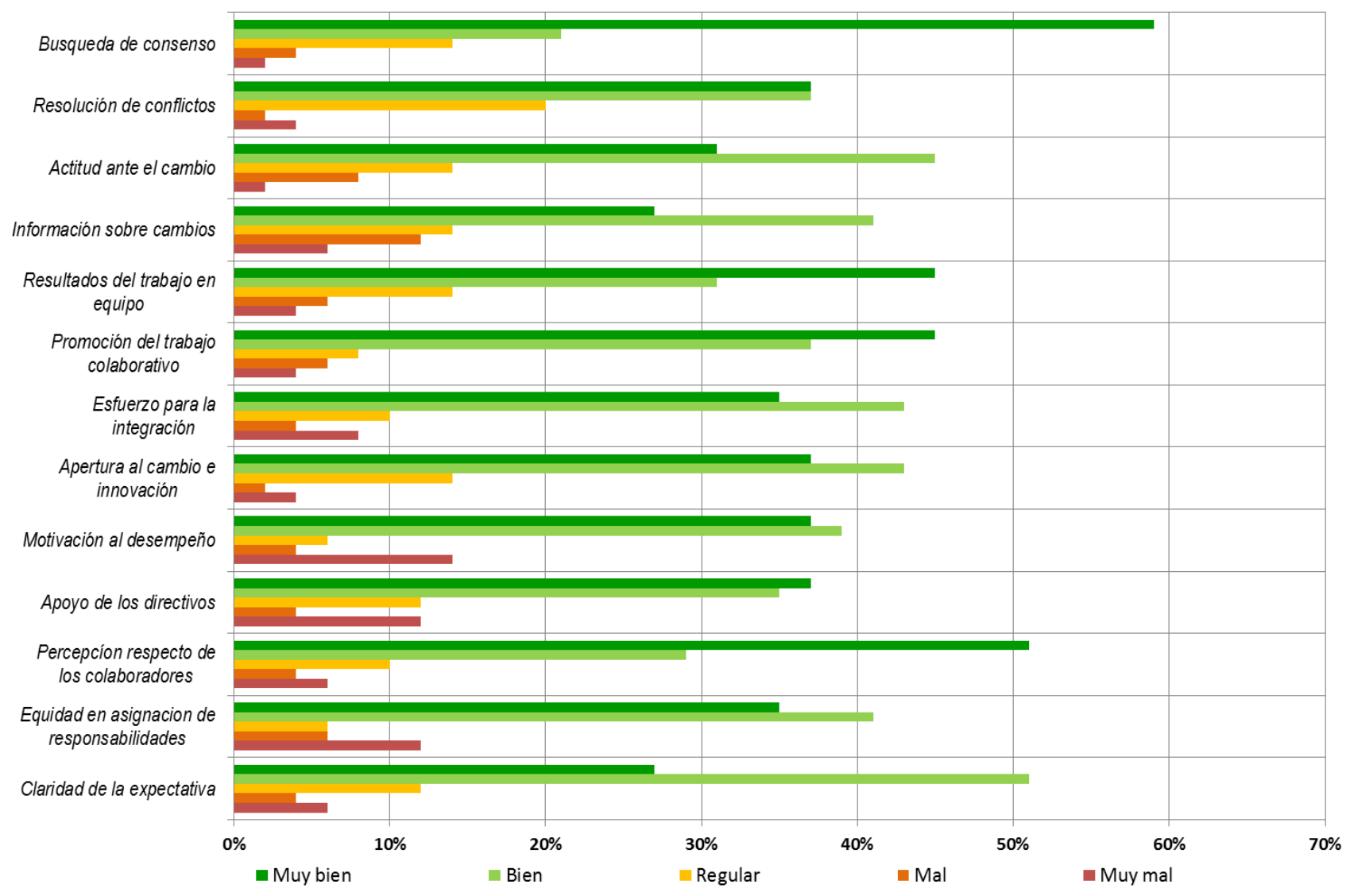

Fuente: elaboración propia, 2013

\section{Motivación, reconocimiento y estímulos.}

En esta categoría se destaca la información de los mecanismos con los que cuenta la institución para motivar, reconocer y estimular el desempeño de los colaboradores, incluyendo los aspectos relacionados con el sueldo y las 
Implicaciones de la cultura organizacional de instituciones de educación superior en la implantación de sistemas de gestión de la calidad con responsabilidad social

Julio Márquez Rodríguez, Sergio Ochoa Jiménez, Beatriz Ochoa Silva

prestaciones, ya que están vinculados estrechamente con la satisfacción y motivación para el trabajo.

En este sentido, se identifican grandes deficiencias en el ámbito del reconocimiento y de la motivación, pero la mayor demanda se orienta hacia las prestaciones laborales. Esto representa una grave debilidad en la búsqueda de un mejor desempeño organizacional y tendrá que considerarse especialmente durante la construcción de estrategias para favorecer mejores condiciones de desempeño.

Figura 6

Resultados de la categoría Motivación, reconocimiento y estímulos

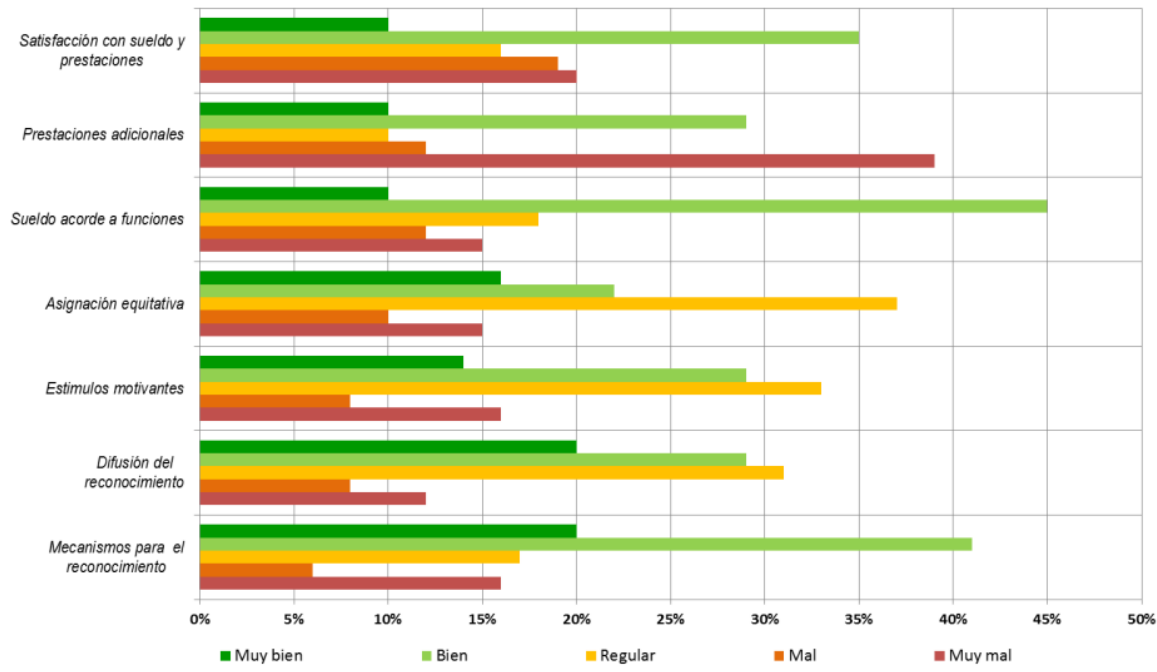

Fuente: elaboración propia, 2013

\section{Normatividad interna.}

En cuanto a la evaluación de la normatividad interna, en el marco de la cultura organizacional, se identificó que su fortaleza radica en ser pública y de fácil acceso para todos los colaboradores, sin embargo se observan debilidades en 
Implicaciones de la cultura organizacional de instituciones de educación superior en la implantación de sistemas de gestión de la calidad con responsabilidad social

Julio Márquez Rodríguez, Sergio Ochoa Jiménez, Beatriz Ochoa Silva

cuanto a su aplicación permanente, así como en el desarrollo de procedimientos para guiar las funciones asignadas.

Cabe destacar que en cuanto al conocimiento, a la claridad y la aplicación del código de ética institucional es posible observar que existen áreas de oportunidad que deberán atenderse para permear este importante referente en el grupo de colaboradores.

Figura 7

Resultados de la categoría Normatividad interna

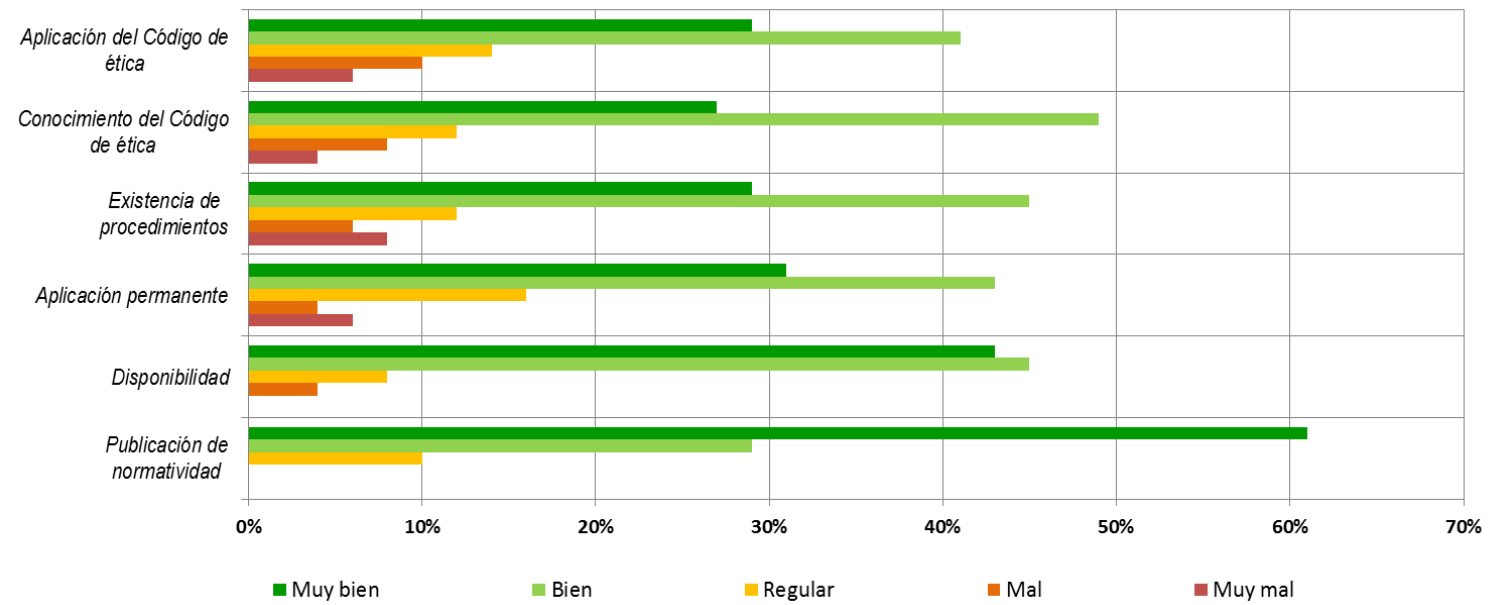

Fuente: elaboración propia, 2013

\section{Capacitación y desarrollo.}

Las áreas de mayor debilidad en esta categoría son la existencia de un plan de desarrollo profesional y la promoción como tal de las iniciativas de desarrollo de los colaboradores, lo cual representa una importante oportunidad de cara a la generación de estrategias de mejora del desempeño organizacional. 


\section{Figura 8 \\ Resultados de la categoría Capacitación y desarrollo}

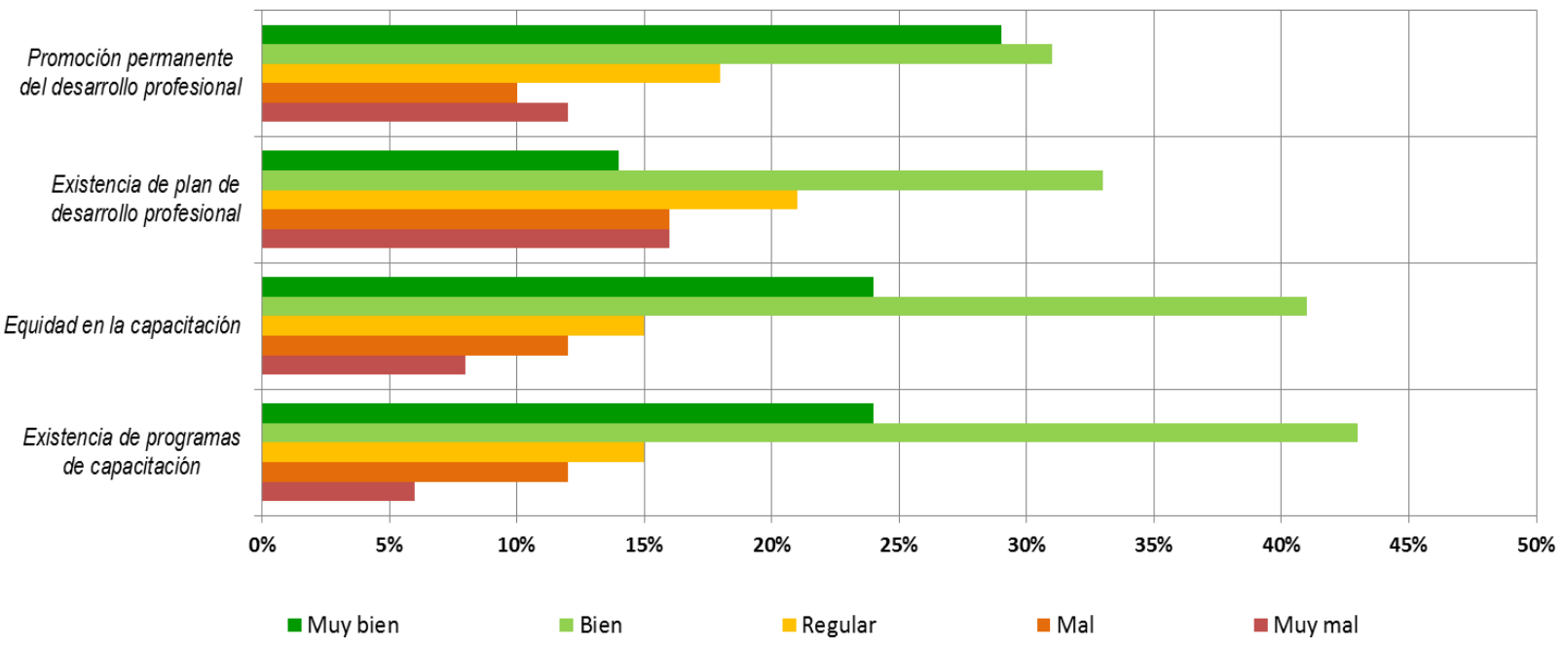

Fuente: elaboración propia, 2013

\section{Actitudes para el trabajo.}

A partir de la aplicación de los instrumentos de recopilación de datos fue posible identificar los principales factores que limitan y que favorecen el desempeño en la IES que se encuentra implementando un sistema de gestión de calidad con responsabilidad social, los cuales se consignan a continuación:

Tabla 2

\section{Factores que limitan el desempeño}

\section{Factores que limitan el desempeño}

- La renuencia al cambio de pensamiento y la mala actitud ante las propuestas de mejora de algunos colaboradores

- La falta de mobiliario y equipamiento suficientes

- El no reconocer las capacidades, las habilidades y los conocimientos

- La falta de retroalimentación
- La dificultad en el acceso a la información

- Falta de mayor integración de personal y de trabajo en equipo

- No contar con los instrumentos y el material necesario para el trabajo

- La falta de prestaciones sociales

- La falta de instalaciones adecuadas para algunos 
Implicaciones de la cultura organizacional de instituciones de educación superior en la implantación de sistemas de gestión de la calidad con responsabilidad social

Julio Márquez Rodríguez, Sergio Ochoa Jiménez, Beatriz Ochoa Silva

- La falta de comunicación clara, precisa y oportuna

- Las deficiencias en el servicio de Internet

- La falta de congruencia y objetividad en la asignación de tareas

- La falta de recursos para prácticas y de apoyo a proyectos

- La falta de becas e incentivos para la investigación

- Pocas acciones para mejorar la salud del personal

\section{trabajos}

- Existencia de trámites y procesos largos y tediosos

- No tener bien definidas las funciones a desempeñar

- La inequidad en la asignación de actividades

- La falta de capacitación en algunos rubros

- La falta de planificación en las actividades

Tabla 3

Factores que favorecen el desempeño.

\section{Factores que favorecen el desempeño}

- La disposición de toda la comunidad universitaria hacia la mejora

- El apoyo de los directivos

- La disposición de recursos e instalaciones

- El trabajo en equipo, la confianza y la armonía

- La retroalimentación periódica de los trabajadores y la integración

- La objetividad, la congruencia y la verdad

- El fomento de la equidad e imparcialidad

- La buena comunicación

- La actitud positiva por parte del personal

- El respaldo y apoyo brindado por los superiores

- El acceso a la información de los medios
- El horario flexible

- El buen ambiente entre colaboradores

- El sueldo acorde al mercado

- El compromiso de la institución hacia la mejora

- La promoción de estímulos, la capacitación y el buen ambiente de trabajo

- Que exista congruencia entre lo que se exige y los medios que se proveen para su cumplimiento.

- El establecimiento de normatividad clara

- La existencia de sistemas de información

Fuente: elaboración propia, 2013

\section{Conclusiones}

El diagnóstico de la cultura organizacional de una IES constituye un proceso de análisis que facilita la identificación de la situación que experimenta la organización educativa en un momento determinado, y se ejecuta con el propósito de coadyuvar a la detección de problemáticas relacionadas con el comportamiento de los colaboradores y la mejora del desempeño organizacional. 
Para contar con un modelo de diagnóstico orientado a reconocer de manera general los principales rasgos de la cultura organizacional en una IES mexicana, especialmente aquellos que favorecen o que limitan la implementación de un Sistema de Gestión de la Calidad con responsabilidad social, se analizaron componentes de diferentes modelos orientados a cubrir aspectos específicos que demanda dicha implementación.

El modelo propuesto está integrado por diez variables, compuestas por elementos de análisis que se vinculan con la puesta en marcha de iniciativas de mejora de la calidad, preferentemente en el ámbito educativo.

Tomando en consideración los resultados del estudio de la cultura organizacional, así como los elementos de la misma que favorecen y que dificultan la implementación de un sistema de gestión de calidad con responsabilidad social en una IES, se definió un conjunto de estrategias de ajuste en seis de los elementos más relevantes de la cultura organizacional: 
Implicaciones de la cultura organizacional de instituciones de educación superior en la implantación de sistemas de gestión de la calidad con responsabilidad social

Julio Márquez Rodríguez, Sergio Ochoa Jiménez, Beatriz Ochoa Silva

\section{Tabla 4 \\ Conjunto de estrategia de ajuste en seis de los elementos más relevantes de la cultura organizacional}

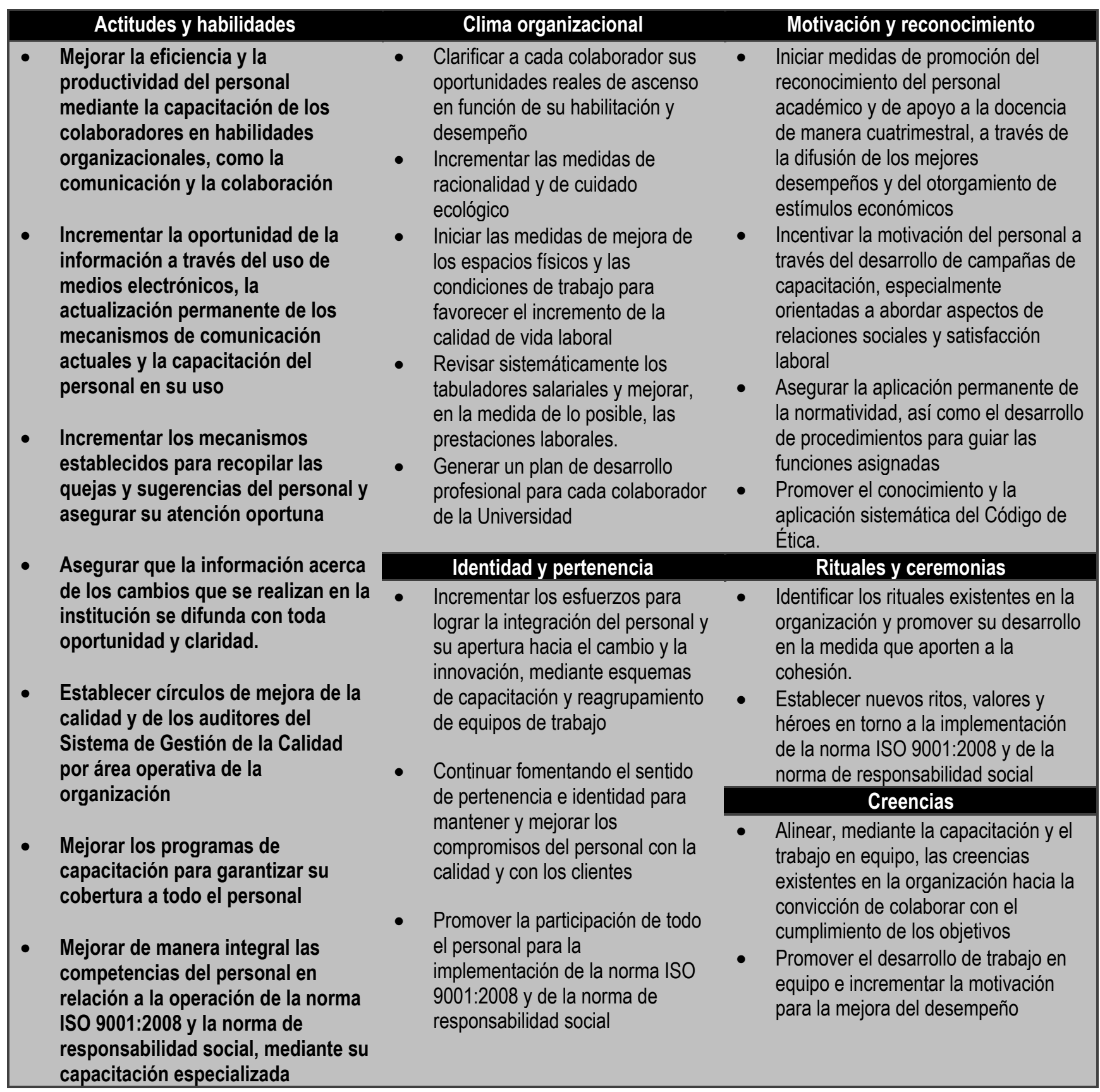

Fuente: elaboración propia, 2013

Revista CAES Vol. 4, No. 2, Año 2013 ISSN-1659-4703 
Mediante el desarrollo del presente estudio fue posible analizar un conjunto de factores de la cultura organizacional de una IES, con la finalidad de lograr identificar aquellos elementos culturales que favorecen o que limitan la implementación de un sistema de gestión de calidad con responsabilidad social, y en función de su identificación proponer un conjunto de estrategias de ajuste de dichos elementos culturales.

Con los resultados identificados es posible comprender mejor los elementos de la cultura organizacional y gestionarlos para propiciar las condiciones que contribuyan a lograr un mejor desempeño de la organización, que favorezca la consecución de los objetivos institucionales, el cumplimiento de su misión y el logro de su visión.

Mediante el desarrollo del conjunto de estrategias y actividades de ajuste a los elementos de la cultura organizacional es posible contribuir de manera sustantiva al logro exitoso de la implementación de un Sistema de Gestión de la Calidad con responsabilidad social, en un menor plazo y con mejores condiciones para su mantenimiento y operación.

\section{Referencias bibliográficas}

Aguilar, A. (2006). El Diagnóstico de la Cultura Organizacional o las culturas de la cultura. Global Media Journal Edición Iberoamericana, 6(11), 67-81.

Barrera, E. (2007). La empresa social y su responsabilidad social. Innovar: Organizaciones y Cultura, 17(30), 59-76.

Buenaventura, P. (1995). Dirigir el cambio de la cultura organizacional hacia la calidad total. Cuadernos de Ciencias Económicas y Empresariales, 28,1118.

Fernández, J. (2002). Cultura de la organización y centro educativo (Memoria para optar al grado de Doctor).Universidad Complutense de Madrid, España. 
Implicaciones de la cultura organizacional de instituciones de educación superior en la implantación de sistemas de gestión de la calidad con responsabilidad social

Julio Márquez Rodríguez, Sergio Ochoa Jiménez, Beatriz Ochoa Silva

Garmendia, J. A. (1990). Desarrollo de la organización y cultura de la empresa. España: ESIC.

Hernández, R., Fernández, C. y Baptista, P. (2008). Metodología de la Investigación ( $4^{\mathrm{a}}$ ed.). México: Mc Graw-Hill.

Hernández, V., Quintana, L., Guedes, R., Mederos, R. y Sablón, N. (2007). Estudio de la motivación, la satisfacción laboral, el liderazgo, la cultura organizacional y la calidad del servicio existente en el Hospital Mario Muñoz Monroy. Folletos gerenciales. Centro Coordinador de Estudios de Dirección, España.

Hellriegel, D. y Slocum, J. (2009). Comportamiento Organizacional (12 $2^{\underline{a}}$ ed.). México: Cengage.

Martínez, E. y Robles, C. (2009). Cultura organizacional en el sistema de gestión de calidad en las dependencias de educación superior Valle de México. Revista Gestión y Estrategia, 2(36), 54-65.

Maull, R., Brown, P. y Cliffe, R. (2001). Organizational culture and quality improvement. International Journal of Operations \& Production Management, 21(3), 302-326.

Montes, F. (2012). Liderazgo y clima organizacional como agentes constructores de una cultura organizacional en instituciones de nivel superior. Visión Educativa, 6(13), 43-52.

Robbins, S. y Judge, T. (2009). Comportamiento Organizacional (13 ${ }^{\mathrm{a}}$ ed.). México: Pearson Educación.

Schein, E. (2010). Organizational Culture and Leadership (4th Ed.). USA: Wiley.

Toca, C. y Carrillo, J. (2009). Asuntos teóricos y metodológicos de la cultura organizacional. Revista Civilizar, 9(17), 117-136. 